\title{
Comparación de los efectos en tratamientos de autismo a partir de modelos conductuales y psicoanalíticos
}

\section{Comparison of the effects in autism treatments from behavioral and psychoanalytic models}

ORIARD-VALLE, Michel ${ }^{1} \dagger^{*}$, JUÁREZ-CAMPUSANO, Yara Suhan ${ }^{1}$ y JIMENEZ-BARRIOS, Jearim Isai $^{2}$

${ }^{1}$ Universidad Tecnológica de México (UNITEC), Campus Querétaro, Av. 5 de Febrero 1412, San Pablo, 76130 Santiago de Querétaro, Qro

${ }^{2}$ Universidad Autónoma de Querétaro, Centro Universitario, Cerro de las Campanas s/n C.P. 76010, Cto Universitario, Centro Universitario, 76010 Santiago de Querétaro, Qro.

ID $1^{\text {er }}$ Autor: Michel, Oriard-Valle / ORC ID: 0000-0002-1186-0941, Researcher ID Thomson: S-5919-2018, CVU CONACYT ID: 781986

ID $1^{\text {er }}$ Coautor: Yara Suhan, Juárez-Campusano / ORC ID: 0000-0003-0886-5466, CVU CONACYT ID: 710264

ID $2^{\text {do }}$ Coautor: Jearim Isai, Jimenez-Barrios / ORC ID: 0000-0003-1209-7391, CVU CONACYT ID: 1013582

DOI: $10.35429 / \mathrm{JCP} .2019 .8 .3 .17 .21$

Recibido 03 de Marzo, 2019; Aceptado 30 de Junio, 2019

\section{Resumen}

En el presente trabajo fueron revisados los resultados reportados en los últimos seis años sobre la eficiencia de tratamientos de autismo a partir de modelos conductuales y psicoanalíticos. Dado que ambos modelos parten de concepciones distintas sobre la dirección del tratamiento en el autismo, se cotejaron los resultados entre sí para determinar sus diferencias generales. Los modelos psicoanalíticos tienen múltiples acepciones del autismo, algunos lo consideran como parte de las psicosis y otros lo ubican como una estructura clínica diferente. Sin embargo, de manera general parten de condiciones simbólicas y de la historia familiar para centrarse en favorecer las interacciones sociales afectivas y el reconocimiento de la alteridad. Mientras que los modelos conductuales parten de condiciones ambientales, genéticas y sociales para disminuir la manifestación de conductas auto-lesivas a partir de recompensas sociales e impactar sobre el contexto familiar gracias al desarrollo socioemocional. Coincidentemente tanto los modelos psicoanalíticos como los conductuales procuran incidir sobre la angustia presente en los pacientes y en sus familiares.

Autismo, Psicoanálisis, Modelo conductual

\begin{abstract}
In this paper we reviewed the autism treatments efficiency results based on behavioral and psychoanalytic models reported within the last six years. Since both models are based on different conceptions of treatment direction in autism cases, the results were compared between them to determinate their general differences. Psychoanalytic models have multiple autism meanings, some of these theories consider it as part of psychoses while others think about it as a different and autonomous clinical structure. Nonetheless, generally these models are based on symbolic conditions and family history to focus treatment on affective social interactions and alterity acknowledge. Meanwhile, behavioral models are based on environmental, genetic and social conditions to decrease the presence of self-injurious behavior through social rewards during therapy and to impact the family context thanks to socioemotional development. Coincidentally psychoanalytic and behavioral models ensure in their interventions to modify the presence of angst in patients and in their relatives.
\end{abstract}

Autism, Psychoanalysis, Behavioral model

Citación: ORIARD-VALLE, Michel, JUÁREZ-CAMPUSANO, Yara Suhan y JIMENEZ-BARRIOS, Jearim Isai. Comparación de los efectos en tratamientos de autismo a partir de modelos conductuales y psicoanalíticos. Revista de Pedagogía Crítica. 2019, 3-8: 17-21

\footnotetext{
* Correspondencia del Autor (orvami@ outlook.com)

$\dagger$ Investigador contribuyendo como primer autor.
} 


\section{Introducción}

El autismo es conceptualizado de diferentes maneras dependiendo del contexto en el cual se estudie, adoptando así las perspectivas de hombre y su cuerpo, las nociones del ser, la educación, entre otras. (Corredera González \& de Battista, 2016). Simultáneamente se ha establecido la noción del autismo como un trastorno psico-neuro-biológico que se presenta en la infancia. Al respecto, se han clasificado seis tipos de intervenciones con el autismo: biomédicas, psicoeducativas, conductuales, conductuales, evolutivas y basadas en terapias (Pérez, \& Pérez, 2018). Dividiendo los tratamientos en dos: educativos y terapéuticos. Esencialmente el enfoque conductual es tomado como el fundamento de los primeros y el enfoque psicoanalítico para los segundos. Vale aclarar que ninguna propuesta de tratamiento asevera curar el autismo.

El autismo comprende una disfunción neuropsiquiátrica que se muestra altamente heredable y observable desde la temprana infancia. La causa es desconocida (Xu et al., 2018), empero se le atribuye a un posible factor extra uterino o ambiental que interactúa con la susceptibilidad genética (López et al., 2018). Para el diagnóstico se toman en cuenta características englobadas en tres grupos (Martelo-Ortiz, Cohen \& Soto-Cantero, 2018) denominados como alteraciones en las relaciones sociales, en la comunicación y comportamientos, intereses y actividades estereotipadas y rígidas -a estas últimas se les da preponderancia, específicamente al estar relacionadas con la agresión- (Hervás \& Rueda, 2018).

La etiología del autismo representa una controversia; sin embargo, comprende factores como alteraciones neurológicas funcionales y estructurales de origen genético y epigenético con base en los datos recabados por el Centro para el Control y Prevención de Enfermedades de EE.UU. y al estudio realizado por la Red Autism and Developmental Disabilities Monitoring (ADDM) (Sanz, Tárraga \& Lacruz, 2018). Al no existir marcadores biológicos, el diagnóstico se fundamenta en el juicio clínico (Reynoso, Rangel \& Melgar, 2017) y el uso de recursos farmacológicos complementarios es recomendado ante conductas agresivas (Hervás \& Rueda, 2018).
El objetivo del presente trabajo es contrastar los resultados reportados de tratamientos conductuales y tratamientos psicoanalíticos para discernir sus diferencias y semejanzas. Por lo tanto se describe de manera general qué se entiende por autismo en los modelos conductuales y en los psicoanalíticos; así como las condiciones para iniciar un tratamiento y sus características principales. Después los resultados son englobados y discutidos.

\section{Autismo en modelos conductuales}

Para los modelos conductuales, el autismo es un trastorno mental nominado Trastorno del Espectro Autista (TEA). Este es considerado una alteración en la adquisición de habilidades socioemocionales durante el desarrollo temprano que a su vez detona una incapacidad progresiva para la adaptación social, así como conductas repetitivas y que persiste durante toda la vida del individuo (Rascón, Del Corral \& Palazón, 2015; Reynoso, Rangel \& Melgar, 2017).

El tratamiento requiere de intervención médica y terapéutica intensiva, abarcando la crianza-educación y el contexto familiar (Zalaquett et al., 2015). Una conducta a la vez es trabajada contemplando metas, y los fármacos son empleados en conductas impulsivas, falta de atención y convulsiones (Reynoso, Rangel \& Melgar, 2017).

Los métodos más empleados son: EIBI, en la atención temprana específicamente; ABA, Pivotal Response Treatment, Early Start Denver Model, TEACHH, STAR, PECS, las historias sociales y la intervención mediada por pares (Andreadi, Charitaki \& Soulis, 2018; Reynoso, Rangel \& Melgar, 2017; Sanz, Tárraga \& Lacruz, 2018).

\section{Autismo en modelos psicoanalíticos}

Dentro de la diversidad de acepciones en los modelos psicoanalíticos, el autismo es planteado como una forma de psicosis o como una posición subjetiva caracterizada por la inexistencia del gran Otro (Laurent, 2013; Tendlarz \& Alvarez Bayón, 2013). 
Alexandre Stevens (citado por Cucagna \& Rost, 2018) propuso que el tratamiento conlleva "ponerse al servicio" del autista, entendiendo su lógica y siguiendo sus intereses, vale decir que es un acompañamiento. La presentación del analista sería como Otro no invasivo. Las intervenciones están encaminadas a que el paciente pueda hablar de lo que le sucede.

Maleval (citado por Sosa, 2017) menciona que desde la perspectiva postfreudiana, se ve al autismo como una patología severa que estaría destinada a mitigar una angustia grave. Por su parte la "orientación lacaniana" plantea al autismo como un "[...] funcionamiento subjetivo singular" (Sosa, 2017 p. 321), contraponiéndose a la perspectiva de una causa en el neurodesarrollo. Proponiendo un modo de trabajo que se base en la revisión de testimonios de otros autistas, considerando la transferencia como motor de cura, esto encaminado a una dirección del tratamiento que consista en la inserción de un otro que no se vuelva intruso y a retomar las propias invenciones e intereses del niño sin desdeñarlas.

A su vez, Bettelheim formuló que la causa del autismo es una vivencia terrible y desesperanzadora. Tustin planteó que también es una vivencia catastrófica de la no satisfacción mutua entre el niño y su madre la que provocaría el autismo. Meltzer por su parte desarrolló una noción previa al estado esquizo-paranoide de Melanie Klein, proponiendo un desmantelamiento en el que el autista se encontraría en una inactividad mental. Estos tres psicoanalistas coinciden en la noción de desarrollo y evidentemente el autismo se presentaría como un problema en aquél. Asimismo los autores sugirieron un fin (o suspensión del tratamiento) satisfactorio en la medida que varias de las conductas se habían transformado (Basualdo, Sosa \& Gascón, 2017).

La disminución de la angustia en el autista y sus familiares, aunado a la posibilidad de un mayor contacto social, son reportados como resultados de los tratamientos psicoanalíticos (Tendlarz, 2016).

\section{Metodología a desarrollar}

Contraste de los resultados reportados en los últimos seis años sobre la eficiencia de tratamientos de autismo a partir de modelos conductuales y psicoanalíticos.

ISSN 2523-2479

ECORFAN $^{\circledR}$ Todos los derechos reservados

\section{Resultados}

Los modelos conductuales para el tratamiento del autismo son los que han presentado mayor evidencia empírica a su favor en la comunicación y las habilidades sociales. La terapia ocupacional con integración sensorial ha demostrado evidencia de efectividad y eficacia en pruebas aleatorizadas. Por otra parte, existe un pronóstico incierto dentro de cualquier tratamiento ya que puede suceder que el niño ni siquiera responda ante este, volviendo el pronóstico impredecible (Vergara, Sosa, Pino, Ortiz \& Hidalgo, 2018).

Los resultados reportados de los modelos psicoanalíticos señalan cambios en los vínculos sociales de la persona diagnosticada con autismo, observando una mayor tolerancia al contacto y la percepción de la presencia del psicoanalista y de los familiares formulada como una manera de reconocer la alteridad. Empero, estos resultados no formalizan indicadores exentos de la opinión de quienes condujeron los tratamientos; representando esto último un sesgo sobre la garantía de que los efectos fuesen por el tratamiento.

\section{Agradecimiento}

Agradecemos a la Unidad de Atención Psicoanalítica Interdisciplinaria (UAPI) de la Universidad Autónoma de Querétaro y a la Universidad Tecnológica de México por su apoyo al desarrollo del presente trabajo de investigación.

\section{Conclusiones}

Los trabajos basados en modelos conductuales plantean la existencia de daño neuronal, pensado este como irreversible no resultaría posible cambiarlo. Simplemente se podría plantear tratarlo, aminorar los síntomas o su injerencia en la vida de las personas que padecen autismo (Esteban \& Marín, 2018). En otras palabras, el niño autista nunca deja de ser autista.

El autismo parece presentarse como un impasse del tratamiento psicoanalítico. Debido a la coincidencia de parámetros retomados para definir al autismo en modelos conductuales y en los psicoanalíticos los resultados de ambas maneras de intervención son similares al esperar elaboraciones propias del autista y que estas deben ser fomentadas. 
Lo cual representa una contradicción dadas las concepciones de sufrimiento, malestar y ser humano que los modelos emplean en sus desarrollos teóricos y epistemológicos. Entonces resulta pertinente pensar en la forma en que el psicoanálisis puede intervenir sobre el autismo y cómo se podría dar cuenta de la eficacia de este tratamiento sin retomar los parámetros de disciplinas que tienen supuestos teóricos y prácticos enteramente distintos.

Por otra parte, tampoco desde los textos revisados se llega a un planteamiento de la causa del autismo en la que se concuerde y sobre la cual trabajar. De ahí que la sintomatología se haya vuelto, de manera general, el blanco de las intervenciones. No obstante, sí es frecuente encontrar en autores que se nombran de "orientación psicoanalítica", la postura de que el autismo es una posición del sujeto, a la vez que se presenta una relación complicada (o inexistente) con el Otro, afirmación que no presenta más sustento que la falta de habla en los niños.

\section{Referencias}

Andreadi, R., Charitaki, G., \& Soulis, S-G. (2018). The effect of Applied Behavioral Analysis on the communication skills in children with Autism Spectrum Disorder: perceptions of special educators and psychologists. Scolars journal of arts, humanities and social sciences, 6(6), pp. 1218-1228.

Basualdo, A. Sosa, M., \& Gascón, M. (2017). Aportes teórico-clínicos del psicoanálisis posfreudiano a la conceptualización del Autismo: Bettelheim, Tustin y Meltzer. En Piro, M. (Ed.). El autismo perspectivas teóricoclínicas y desafíos contemporáneos (pp. 52-78). Buenos Aires: Editorial de la Universidad de la Plata.

Cucagna, A., \& Rost, G. (2018). Conversando con Alexandre Stevens. Diagnosis, 15(1).

Corredera González, M., \& De Battista, J. L. (2016). Abordajes educativos y terapéuticos sobre las dificultades en la comunicación de niños con signos clínicos de autismo. Acta, 62 (3), 196- 206.
Esteban, Y. A., \& Marín, F. A. (2018). Un modelo de indicador para el seguimiento $\mathrm{y}$ monitorización de la evolución de los Trastornos del Espectro del Autismo. En Intervención en contextos clínicos y de la salud: Volumen II (pp. 105-115). ASUNIVEP.

Hervás, A., \& Rueda, I. (2018). Alteraciones de la conducta en los trastornos del espectro autista. Rev Neurol, 66(Supl 1), S31-8.

Laurent, E. (2013). La batalla del autismo: De la clínica a la política. Buenos Aires: Grama Ediciones.

López, J., De Jesús, G., Bravo, G., Ramos, M., \& Ríos, N. (2018). Autismo y personalidades con trastornos de la conducta autista. Panorama, 14 (1), 53-61.

Martelo-Ortiz, O. M., Cohen, R. J., \& SotoCantero, S. P. (2018). Guía práctica para la evaluación neuropsicológica de pacientes con trastorno del espectro autista (TEA). Consultado en: https://repository.ucc.edu.co/handle/20.500.124 94/12452

Pérez, M. D. L. L. A., \& Pérez, R. B. (2018). Alternativas de tratamiento en los trastornos del espectro autista: una revisión bibliográfica entre 2000 y 2016. Revista de Psicología Clínica con Niños y Adolescentes, 5(1), 22-31.

Rascón, P., R. Del Corral, C., \& Palazón I. (2015). Encopresis en los Trastornos del Espectro Autista: análisis de un caso clínico. Revista de Psicología Clínica con Niños y Adolescentes, 2 (1), pp. 55-61.

Reynoso, C., Rangel, M. J., \& Melgar, V. (2017). El trastorno del espectro autista: aspectos etiológicos, diagnósticos y terapéuticos. Revista Médica del Instituto Mexicano del Seguro Social, 55(2), 214-222.

Sanz, P., Tárraga, R., \& Lacruz, I. (2018). Prácticas psicoeducativas basadas en la evidencia para trabajar con alumnos con TEA. Quaderns digitals: Revista de nuevas tecnologías y sociedad, 87(8), pp. 152-161. 
Sosa, M. (2017). Psicoanálisis y neurociencias. Aportes al complejo campo del autismo infantil. En Psicoanálisis, instituciones de salud y legitimidad epistémica. Conferencia llevada a cabo en el $6^{\circ}$ Congreso Internacional de Investigación de la Facultad de Psicología (UNLP), Provincia de Buenos Aires, Argentina.

Tendlarz, S. E. (2016). Clínica del autismo y de las psicosis en la infancia. Buenos Aires: Colección Diva.

Tendlarz, S. E. \& Alvarez Bayón, P. (2013). ¿Qué es el autismo? Infancia y psicoanálisis. Buenos Aires: Colección Diva.

Vergara, J. M. L., Sosa, A. G. D. J. R., Pino, G. B., Ortiz, M. R., \& Hidalgo, N. P. R. (2018). Autismo y personalidades con trastornos de la conducta autista. Panorama. Cuba y Salud, 14(1).

Xu, W., Zhao, Z., Ceng, M., \& Yao, J. (2018). Efectos de una intervención de un modelo de rehabilitación basado en el ejercicio sobre la soledad y las conductas saludables de adolescentes con autismo. Revista argentina de clínica psicológica, 27(3), 455-465.

Zalaquett, D., Schönstedt, M., Angeli, M., Herrrera, C., \& Moyano, A. (2015). Fundamentos de la intervención temprana en niños con trastornos del espectro autista. Revista Chilena de Pediatría, 86(2), pp. 126-131 